\section{Advantage of diabetes?}

SIR - Non-insulin-dependent diabetes (NIDDM), a familial disorder which develops in middle and later life, has become more common in certain societies such as the Nauruan islanders in Micronesia, where $50-60 \%$ of older adults are affected (see the discussion by Jared Diamond in News and Views ${ }^{1}$ ). It is more prevalent in populations which in the past have endured food scarcity but which now have a sedentary lifestyle, abundant food and frequent obesity. Neel's hypothesis ${ }^{2}$ to explain this high prevalence is that the predisposition to develop NIDDM has been advantageous for survival in the past, possibly because of lower levels of energy expenditure and therefore lower dietary energy requirements. But this hypothesis has been difficult to test because it requires identifying and studying subjects at risk before they develop the disorder.

Gestational diabetes mellitus (GDM) occurs in the third trimester of pregnancy, but usually disappears following delivery. Most women with this condition develop impaired glucose tolerance or NIDDM in later life. The women are also likely to redevelop GDM in a subsequent pregnancy. We have studied post-prandial thermogenesis and resting energy expenditure in women with a previous pregnancy complicated by GDM, when they were pregnant again. They were studied in the second trimester, at which time glucose tolerance was still normal, and once more when glucose tolerance was normal following this later pregnancy. Pregnant women without a personal or family history of diabetes were studied for comparison. Details are reported elsewhere ${ }^{3,4}$.

Post-prandial thermogenesis (also called luxus consumption, the specific dynamic action of protein and dietinduced thermogenesis) is the heat generated following the ingestion of food $^{5}$, representing 14-20 per cent of total daily energy expenditure. In normal pregnancy (see figure), post-prandial thermogenesis decreases, resulting in an energy saving of 39 MJ over the second and third trimesters, or 13 per cent of the additional energy cost of pregnancy. In women with previous GDM, post-prandial thermogenesis decreases further, increasing the energy saving to 22 per cent. Postprandial thermogenesis remains lower in women with previous GDM than in the women who had not had diabetes during pregnancy. In the non-pregnant state this energy saving amounts to $70 \mathrm{~kJ}$ per day. Resting energy expenditure increases as expected during pregnancy, but no differences are observed as a result of the diabetic predisposition.

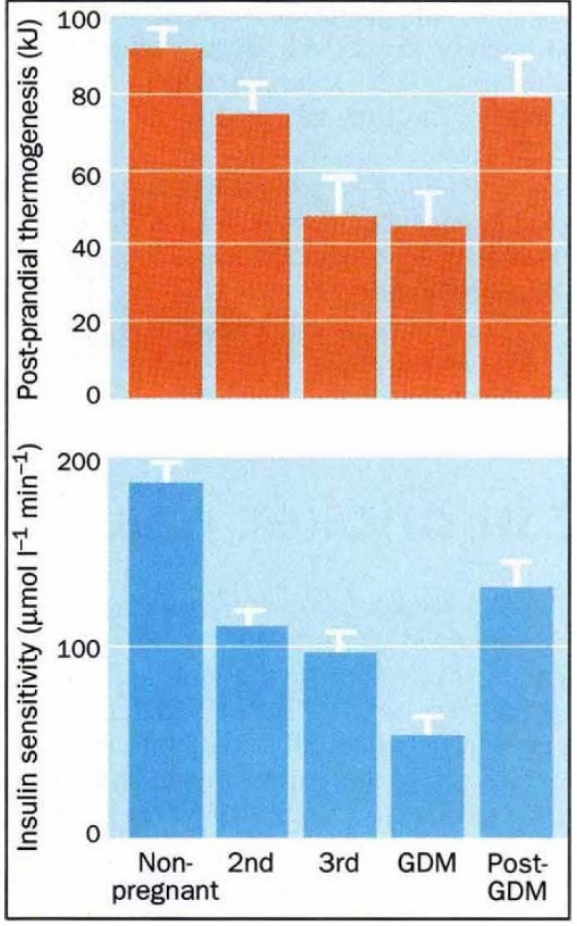

Post-prandial thermogenesis (top) and insulin sensitivity (bottom) (mean s.e.m.) in non-pregnant women, in women during the second (2nd) and third (3rd) trimesters of normal pregnancy, in gestational diabetic women (GDM) studied in the second trimester (before decompensation to diabetes) and in women with GDM studied after delivery when glucose tolerance had returned to normal (post-GDM). Post-prandial thermogenesis was measured by continuous indirect calorimetry following the ingestion of a mixed meal $(42 \mathrm{~kJ}$ per $\mathrm{kg}$ lean body mass). The integrated area of the increment in energy expenditure over resting energy expenditure was calculated and expressed in $\mathrm{kJ}$. Insulin sensitivity was measured with a short insulin tolerance test. The linear slope of the arterialized plasma glucose decline after $0.05 \mathrm{U}$ per $\mathrm{kg}$ body weight intravenous insulin (3-15 min) was calculated and expressed in $\mu \mathrm{mol}{ }^{-1} \mathrm{~min}^{-1}$. Results for post-prandial thermogenesis were $P<0.05$ both in GDM compared with 2 nd trimester women and in post-GDM compared with non-pregnant women, while respective results for insulin sensitivity were $P<0.01$. The data are modified, with additional data, from refs 3 and 4 .

Post-prandial thermogenesis has an obligatory component (reflecting the energy costs of the digestion, absorption, interconversions and storage of food) and a possible facultative component (which has been proposed to modulate energy expenditure). Insulin action is thought to be important in its production. Thus, thermogenesis after eating is reduced in cafeteria-fed diabetic rats and is restored by insulin treatment ${ }^{6}$. In humans, thermogenesis in response to oral glucose is reduced in insulin-resistant states such as obesity, and is reduced further when obese subjects have impaired glucose tolerance? The thermogenic response to insulin and glucose given intravenously is also reduced in obesity and reduced further when insulin sensitivity is lower still in obese subjects with impaired glucose tolerance or diabetes mellitus ${ }^{8}$.

We found that normal pregnancy is associated with a decline in insulin sensitivity (see figure). In this situation, insulin sensitivity correlates positively with post-prandial thermogenesis, again suggesting that these parameters are linked. In the women with previous GDM, insulin sensitivity is reduced further and the reduction persists after delivery. Insulin sensitivity is impaired in other subjects predisposed to NIDDM, such as first-degree relatives of NIDDM patients $^{9}$ (30-40 per cent lifetime risk), although their thermogenesis has not been studied.

The dietary energy saving associated with this insulin resistance in both pregnancy and the non-pregnant state is the evolutionary advantage proposed in this extension of the Neel hypothesis. No advantage is implied once GDM or NIDDM have developed. On the contrary, GDM has disadvantages resulting from fetal macrosomia. Dietary energy restriction is the mainstay of treatment and is sufficient in itself in most women. In situations of limited dietary energy availability, GDM is unlikely to develop and the advantage for survival of a lower energy requirement during and after pregnancy would predominate. Similarly, established NIDDM is associated with a twofold increase in mortality, mainly from vascular disease $\mathrm{e}^{10}$, but also it is less likely to develop in situations of limited food intake. (Diabetes was unknown in the Nauru islanders before they adopted a Western lifestyle.) The evolutionary disadvantage may in any case be small, as NIDDM often does not develop until after the reproductive years.

We conclude that NIDDM is therefore adverse to health, although the predisposition to develop it, and the associated insulin resistance, may once have been advantageous in terms of dietary energy requirements.

\section{Stephen Robinson}

\section{Desmond G. Johnston}

Unit of Metabolic Medicine,

St Mary's Hospital Medical School,

Norfolk Place,

Imperial College of Science, Technology and Medicine,

London W2 1PG, UK

1. Diamond, J. M. Nature 357, 362-363 (1992)

2. Neel, J. V. Am. J. hum. Genet. 14, 353-362 (1962)

3. Robinson, S. et al. Diabet. Med. 11, 545-550 (1994)

4. Robinson, S. et al. Diabet. Med. 10, 139-145 (1993)

5. Miller, D. S. \& Mumford, P. Am. J. clin. Nutr. 20, 1212-1222 (1967).

. Rothwell, N. J. \& Stock, M. J. Metabolism 30, 673-678 (1981).

7. Golay, A. et al. Diabetes 31, 1023-1028 (1982)

8. Ravussin, E. et al. J. clin. Invest. 72, 893-902 (1983).

9. Lillioja, S. et al. New Engl. J. Med. 329, 1988-1992 (1993).

10. Panzram, G. Diabetologia 30, 123-131 (1987). 